\title{
Autoimmune retinopathy associated with monoclonal gammopathy of undetermined significance: a case report
}

\author{
Emily A. Eton ${ }^{*}$, Gary Abrams², Naheed W. Khan ${ }^{1}$ and Abigail T. Fahim ${ }^{1}$
}

\begin{abstract}
Background: Monoclonal gammopathy of undetermined significance (MGUS) is a plasma cell dyscrasia and precursor to multiple myeloma. It has known ocular manifestations, but has not previously been shown to have an association with autoimmune retinopathy.

Case presentation: A 57 year-old female presented with 1 year of progressive, bilateral, peripheral vision loss, photopsias, and nyctalopia. Her fundus examination and extensive ancillary testing were concerning for hereditary versus autoimmune retinopathy. The patient was found to have anti-retinal antibodies against carbonic anhydrase II and enolase proteins with a negative genetic retinal dystrophy panel. Malignancy work-up was negative, but the patient was diagnosed with MGUS, a premalignant condition. The patient was treated with immunosuppressive therapies, with rituximab demonstrating the most robust therapeutic response with respect to patient symptoms and ophthalmic testing.

Conclusions: MGUS should be considered as a potential etiology of autoimmune retinopathy in patients without other autoimmune or malignant disease processes. Immunosuppressive therapy may be helpful in limiting disease progression, with rituximab showing efficacy in retinopathy refractory to other agents.
\end{abstract}

Keywords: Autoimmune retinopathy, Monoclonal gammopathy of undetermined significance, Plasma cell dyscrasias

\section{Background}

Monoclonal gammopathy of undetermined significance (MGUS) is a premalignant clonal plasma cell proliferation characterized by the presence of serum M-protein without associated symptoms of end-organ damage as seen with multiple myeloma (hypercalcemia, renal insufficiency, anemia, bony lesions) [1]. MGUS carries with it a risk of progression to malignancy, with $11 \%$ of MGUS patients developing multiple myeloma or another plasma-cell or lymphoid disorder [2]. Ocular manifestations of MGUS are rare but have been described in the literature and include maculopathy with serous macular detachments [3],

\footnotetext{
* Correspondence: etone@med.umich.edu

'Kellogg Eye Center, University of Michigan, 1000 Wall Street, Ann Arbor, MI 48105, USA

Full list of author information is available at the end of the article
}

crystalline keratopathy [4], and copper deposition in Descemet's membrane [5]. We report a novel case of autoimmune retinopathy in a patient with MGUS.

\section{Case presentation}

A 57 year-old female was referred with 1 year of progressive peripheral vision loss. Additional symptoms included 6 months of progressive nyctalopia and 2 months of photopsias. Her ophthalmologic history was notable for nuclear sclerotic cataracts bilaterally and a complete posterior vitreous detachment bilaterally. Medical history was significant for insulin-dependent type 2 diabetes mellitus without retinopathy and hypertension. The patient reported a family ocular history of a brother with glaucoma.

Ophthalmologic exam demonstrated best corrected visual acuity of $20 / 25$ bilaterally. Color vision testing by

\section{$\triangle B M C$}

(c) The Author(s). 2020 Open Access This article is licensed under a Creative Commons Attribution 4.0 International License, which permits use, sharing, adaptation, distribution and reproduction in any medium or format, as long as you give appropriate credit to the original author(s) and the source, provide a link to the Creative Commons licence, and indicate if changes were made. The images or other third party material in this article are included in the article's Creative Commons licence, unless indicated otherwise in a credit line to the material. If material is not included in the article's Creative Commons licence and your intended use is not permitted by statutory regulation or exceeds the permitted use, you will need to obtain permission directly from the copyright holder. To view a copy of this licence, visit http://creativecommons.org/licenses/by/4.0/ The Creative Commons Public Domain Dedication waiver (http://creativecommons.org/publicdomain/zero/1.0/) applies to the data made available in this article, unless otherwise stated in a credit line to the data. 

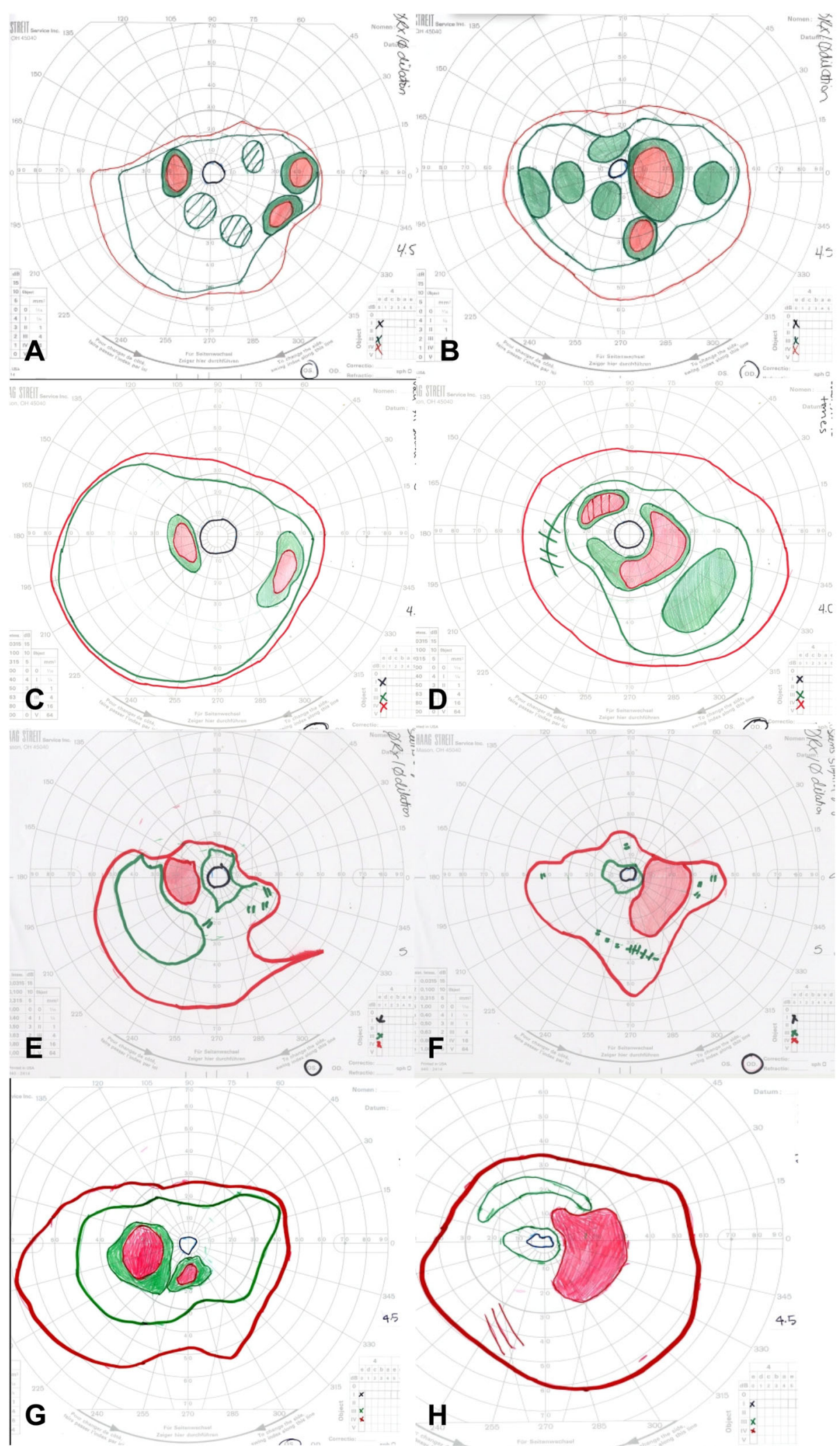

Fig. 1 (See legend on next page.) 
(See figure on previous page.)

Fig. 1 Goldmann visual fields were obtained for targets IV4e (red), III4e (green), and I4e (black). Baseline fields from the left (a) and right (b) eyes showed severe constriction of the I4e isopter and moderate constriction of III4e and IV4e isopters, with enlarged blind spots and scattered midperipheral scotomas bilaterally. Six months later, constriction and scotomas in the left (c) and right (d) eyes were improving on prednisone and methotrexate therapy. Five months after starting methotrexate, there was again worsening constriction of all isopters in the left (e) and right (f) eyes despite increasing methotrexate doses. The IV4e and III4e isopter constriction improved in the left (g) and right (h) eyes following initiation of rituximab infusions

both Ishihara plates and Farnsworth D-15 was without deficits. Goldmann visual fields (GVF) showed moderate to severe constriction bilaterally with enlarged blind spots and scattered mid-peripheral scotomas (Fig. 1a-b). Dark adaptation threshold measured with GoldmannWeekers dark-adaptometer was measured after $45 \mathrm{~min}$ in the dark and was elevated by $1.0 \mathrm{log}$ unit at fixation and $2.5 \log$ units peripherally OD, and $0.7 \log$ units at fixation and $2.5 \log$ units peripherally OS.

Intraocular pressure was $15 \mathrm{mmHg}$ bilaterally with briskly reactive pupils without afferent pupillary defect. Slit lamp exam of the anterior segment was notable for deep and quiet anterior chambers and 1+ nuclear sclerosis bilaterally, and trace anterior vitreous cell in the right eye only. Fundus exam demonstrated Weiss rings bilaterally, mild disc pallor, and severely attenuated retinal vessels with perivascular pigment in both eyes. There was moderate bone spicule pigmentation in the mid-peripheral retina, greater in the right eye than the left eye. (Fig. 2). Macula optical coherence tomography (OCT) demonstrated severe outer retinal atrophy with central sparing of ellipsoid zone and outer nuclear layer bilaterally and trace intraretinal cystic fluid in the right eye (Fig. 3a-c). Fluorescein angiography (FA) was also obtained and in both eyes showed peripheral nonperfusion with late scattered vascular leakage and disc leakage (Fig. 4a-b). Fundus autofluorescence demonstrated diffuse hyperautofluorescence circumferentially in the parafoveal region, bilaterally (Fig. 5). Full-field electroretinography (ERG) performed with a Ganzfeld electroretinogram was recorded using the International
Society for Clinical Electrophysiology of Vision protocol, and was consistent with advanced, symmetric rod-cone degeneration bilaterally (Fig. 6).

Laboratory tests, including antinuclear antibody, antineutrophil cytoplasmic antibody, c-reactive protein, complete blood count, basic metabolic panel, complement $\mathrm{C} 3$, complement $\mathrm{C} 4$, rheumatoid factor, anti-cyclic citrullinated peptide, cryoglobulin, lupus anticoagulant, angiotensin converting enzyme, lysozyme, Ebstein-Barr virus antibody, cytomegalovirus antibody, bartonella antibody, viral hepatitis panel, HIV, lyme antibody, syphilis treponemal screen, and quantiferon gold, were all negative. HLA testing was negative for HLA:A29, B51, and B27 antigens. An anti-retinal autoantibody panel ordered through the ocular immunology laboratory at the Casey Eye Institute was positive for anti-retinal antibodies against $30-\mathrm{kDa}$ carbonic anhydrase II protein and 46-kD enolase protein. Serum protein electrophoresis was performed with an elevated M-spike of $0.38 \mathrm{~g} / \mathrm{dL}$ (IgG lambda subtype). The patient was referred to an oncologist who diagnosed her with MGUS.

There was no family history of retinal dystrophy, and a 267 genetic retinal dystrophy panel through the Ocular Genomics Institute at Massachusetts Eye and Ear Infirmary was inconclusive. Given the relatively rapid progression in conjunction with vascular leakage on FA and the positive anti-retinal antibodies, the clinical picture was consistent with autoimmune retinopathy (AIR). Given concern for possible cancer-associated retinopathy (CAR), oncologic screening was reviewed and included a normal mammogram 9 months prior to presentation,

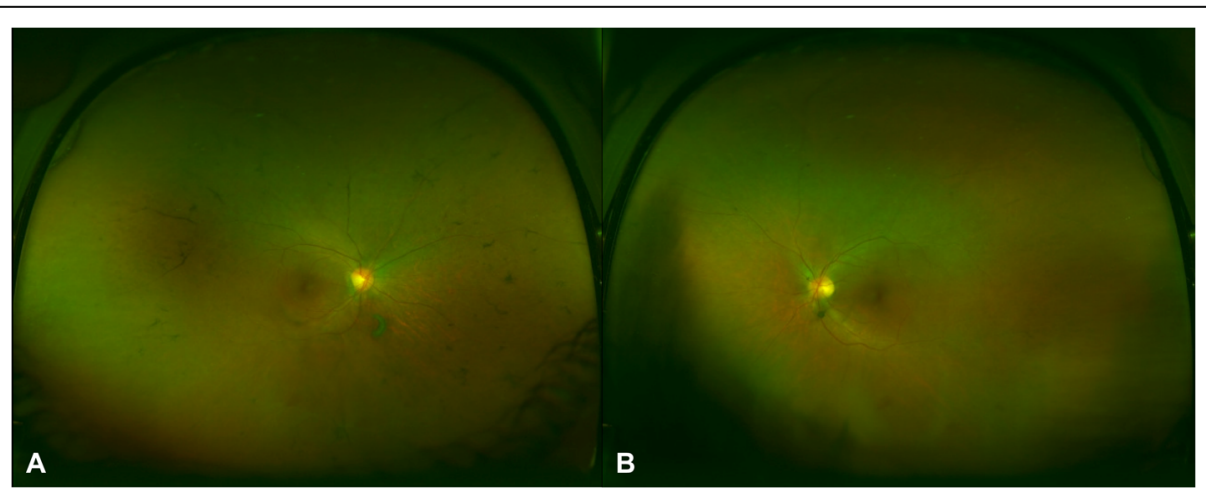

Fig. 2 Ultra-widefield color fundus photos of the right (a) and left (b) eye demonstrated mild optic disc pallor, attenuation of retinal vessels, and mid-peripheral bone spicule pigmentation in the right eye greater than left 


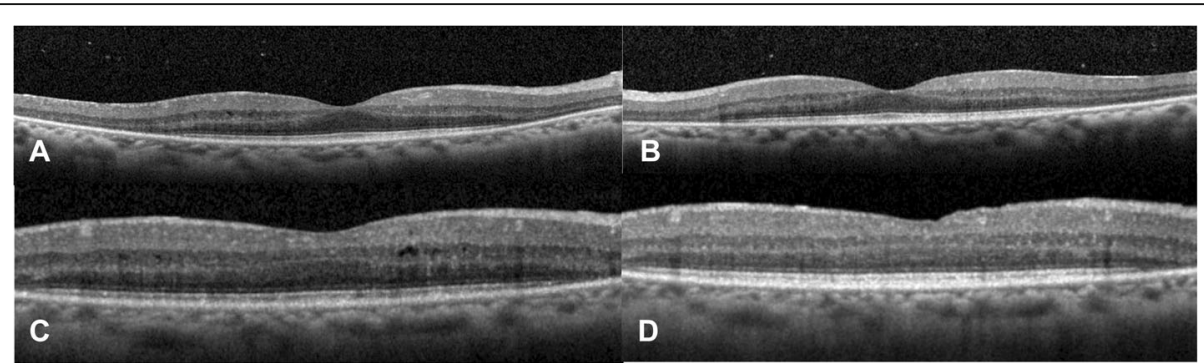

Fig. 3 Macula OCT demonstrated preserved central island of the ellipsoid layer and outer nuclear layer in the right (a) and left (b) eyes, with otherwise severe outer retinal atrophy and trace cystic intraretinal fluid in the right eye (c). Six months later on prednisone and methotrexate therapy there was resolution of intraretinal fluid in the right eye (d)

negative fecal occult blood test 2 months prior to presentation, colonoscopy without malignant lesions 5 years prior, and normal pap smear 1 year prior. CT of the chest and abdomen and MRI of the brain and orbits were also negative for malignancy.

The patient was started on prednisone $70 \mathrm{mg}(1 \mathrm{mg} /$ kg-day) with a slow taper and mycophenolate mofetil $1500 \mathrm{mg}$ twice daily was initiated within the next month. She had multiple episodes of skin peeling, which resolved after discontinuing mycophenolate. Methotrexate $15 \mathrm{mg}$ weekly was initiated and the patient continued tapering prednisone down to $20 \mathrm{mg}$ daily over a period of 6 months. On this regimen, she had improvement in her visual acuity, to 20/20 bilaterally, and GVF (Fig. 1c-d), resolution of macular edema on OCT (Fig. 3d), and decreased vascular leakage on FA (Fig. 4c-d). Her fundus examination remained stable. She was tapered off prednisone completely over another 4 months. One month later on methotrexate monotherapy, the patient was noted to have progressive constriction of GVF in the right eye. Methotrexate was increased to $17.5 \mathrm{mg}$ weekly. Four months later, the visual acuity was $20 / 25$ bilaterally and the patient noted a subjective decrease in peripheral vision in the right eye. Continued bilateral progression on GVF (Fig. 1e-f), return of macular edema bilaterally, and further attenuation of parafoveal ellipsoid zone in the right eye were noted. A single sub-Tenon's methylprednisolone acetate injection $(40 \mathrm{mg})$ in her right eye, the worse eye, was used to bridge the patient to rituximab infusions. After the first two rituximab infusions

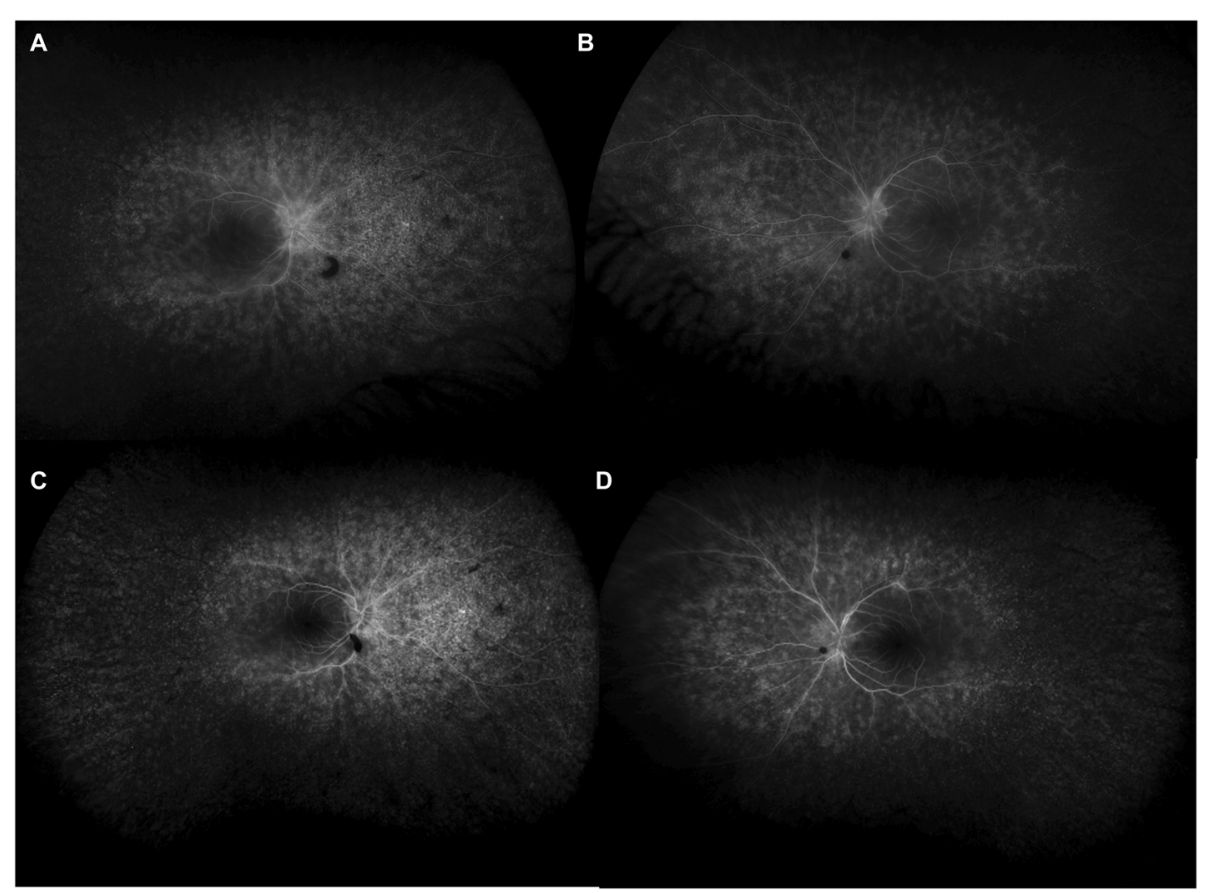

Fig. 4 Late phase fluorescein angiography on initial presentation showed scattered areas of deep leakage with peripheral non-perfusion and disc leakage in the right (a) greater than left (b) eyes. After six months of immunosuppressive therapy there was interval decrease in leakage in the right (c) and left (d) eyes 


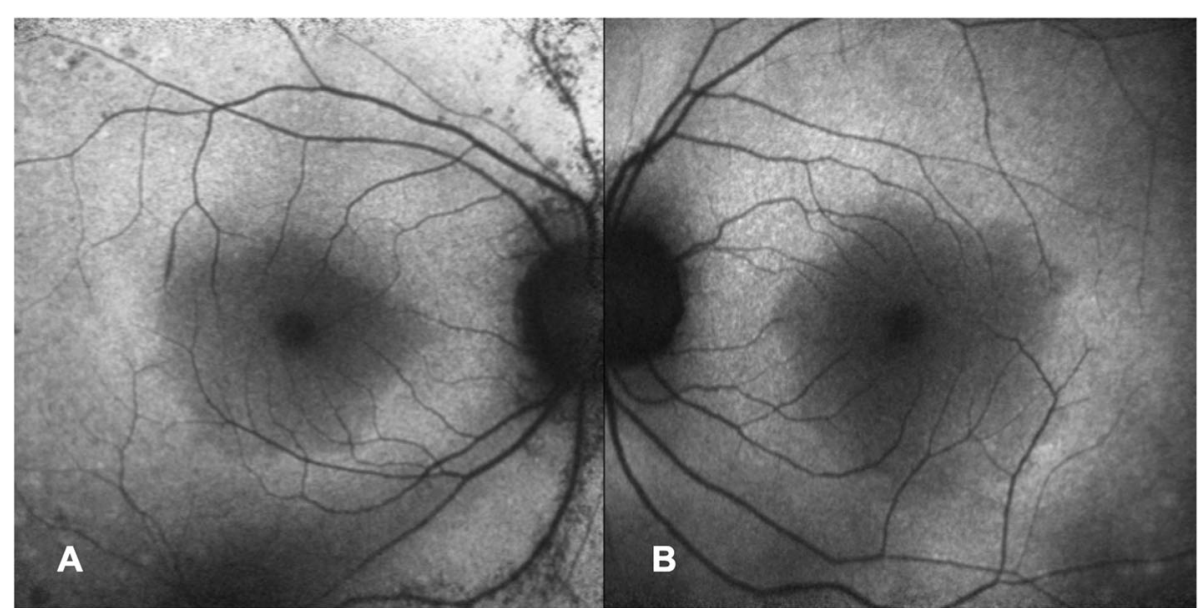

Fig. 5 Fundus autofluorescence showed slightly increased autofluorescence circumferentially in the parafoveal region in the right (a) and left (b) eyes, with hyper- and hypoautofluorescent speckling in the right eye along the superior and inferior arcades

(1000 mg each) there was improvement in GVF constriction (Fig. 1g-h) and resolution of macular edema in both eyes. The visual acuity was 20/30 bilaterally. The patient missed her subsequent scheduled infusions due to transportation issues, and when seen 6 months after her last infusion she had a decrease in visual acuity to $20 / 40-1$ in the right eye and 20/30-1 in the left eye, recurrence of mild cystoid macular edema bilaterally, and enlarging central scotoma in the right eye. She has recently restarted rituximab infusions. Throughout her clinical course her MGUS remained stable.

\section{Discussion and conclusions}

MGUS is a common pre-malignant plasma cell disorder with $3.2 \%$ of patients over the age of 50 and $5.3 \%$ of patients over the age of 70 affected [6]. The disorder is characterized by a monoclonal immunoglobulin (M-protein) elevation of less than $30 \mathrm{~g} / \mathrm{L}$, bone marrow plasma cell involvement of less than 10\%, and lack of sequelae attributable to multiple myeloma (hypercalcemia, renal insufficiency, anemia, or bony lesions). Patients with IgM MGUS tend to progress to Waldenström's macroglobulinemia (WM) whereas those with IgA or IgG MGUS progress to multiple myeloma at a rate of $1 \%$ per year [7]. Our patient had a low burden of M-protein, decreasing her risk of progression to multiple myeloma, however she continues to be followed routinely by oncology with serial monoclonal protein levels.

Ocular manifestations of MGUS have been described [3-5], but to our knowledge, there are no prior reports of MGUS-associated autoimmune retinopathy. AIR is characterized by photoreceptor dysfunction and the

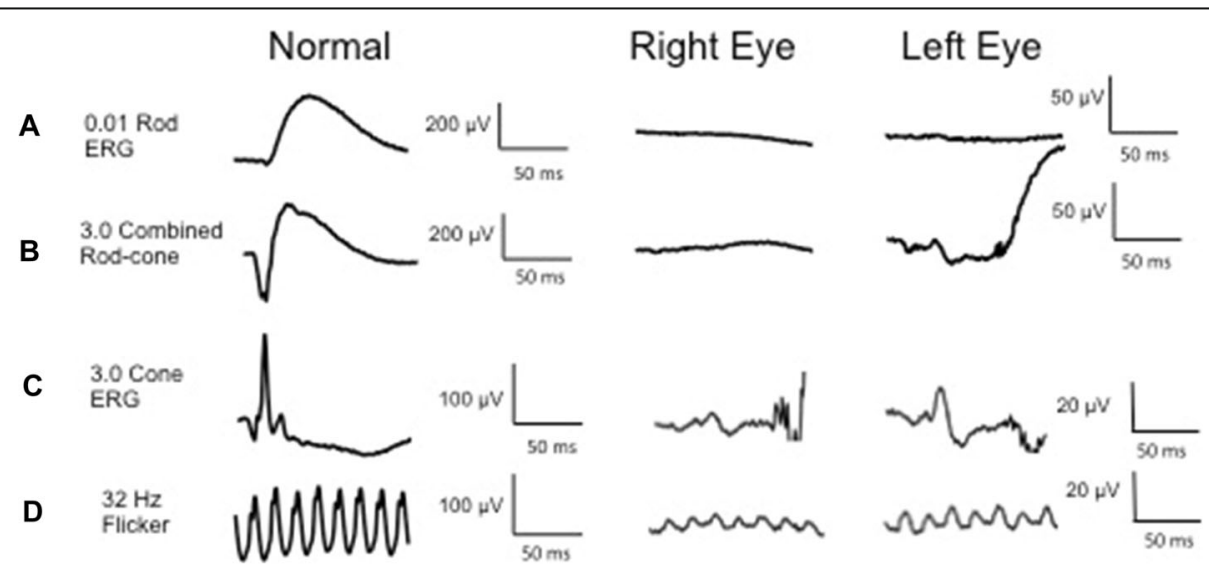

Fig. 6 Full field electroretinography showed a diminished 0.1 rod isolated ERG b-wave bilaterally, consistent with severely decreased rod function; b scotopic 3.0 ERG with depressed combined rod-cone response in the right eye and 10\% of normal a- and b-waves in the left eye; c residual 3.0 ERG cone response with 5\% normal amplitude and delayed peak implicit time bilaterally; d photopic $3.032 \mathrm{~Hz}$ flicker reduced to $10 \%$ normal amplitude and delayed implicit time bilaterally 
presence of anti-retinal autoantibodies, with associated visual symptoms that may include visual field defects, decreased visual acuity, scotomas, nyctalopia, and photopsias. It can be divided into paraneoplastic and nonparaneoplastic disease processes, with the paraneoplastic subset further divided into cancer-associated retinopathy (CAR) and melanoma-associated retinopathy (MAR) $[8,9]$. CAR is most prevalent in association with small-cell lung carcinoma, but has also been seen in gynecologic and breast cancers, other solid tumor malignancies, and hematologic malignancies [10, 11]. Non-paraneoplastic AIR (npAIR) patients are typically females in their 50s-60s, similar to our patient, and often have a family or personal history of autoimmune disease. Our patient reported an unspecified family member with rheumatoid arthritis. Our patient had an extensive workup, which was negative for malignancy but positive for MGUS, a non-malignant neoplastic disorder.

Though MGUS and multiple myeloma have not previously been linked to autoimmune retinopathy, cancerassociated retinopathy has been reported in the setting of WM. Liu et al. described a patient who carried a diagnosis of WM previously treated with plasma exchange, who developed scotomas, photopsias, and visual blurring [12]. Workup demonstrated depressed scotopic and photopic responses on full-field ERG with autoantibodies to $35-\mathrm{kDa}$ glyceraldehyde 3-phosphate dehydrogenase as well as 43- and 44-kDa unspecified retinal proteins. Plasma exchange therapy was successful in stabilizing his cancer-associated retinopathy. Sen et al. described a WM patient who initially presented with cone dysfunction but later developed nyctalopia and showed additional rod dysfunction on ERG [13]. An autoantibody to the connecting cilium present at the inner segment/ outer segment photoreceptor junction was identified on immunohistochemistry. The disease course was notably slower in progression than is often seen with CAR. Rituximab therapy did not result in clinical improvement for this patient. In both cases, the patients carried a WM diagnosis prior to developing visual symptoms, whereas our patient's MGUS was newly identified as part of her retinopathy workup.

While WM and multiple myeloma are considered malignant disorders in contrast to MGUS, which is a nonmalignant neoplastic process, all three disorders share an underlying mechanism of immune dysregulation, aberrant plasma cell proliferation, and resultant clonal antibody production. Immune dysregulation is believed to explain the associations seen between MGUS and a variety of autoimmune disorders, including ankylosing spondylitis, polymyositis, rheumatoid arthritis, polymyalgia rheumatica, pernicious anemia, and demyelinating neuropathy [14]. It stands to reason that dysregulated antibody production may underlie the development of AIR in MGUS patients, although we cannot exclude the possibility that this patient developed AIR independent of MGUS. Various retinal antibodies have been associated with AIR including autoantibodies against recoverin, inner plexiform layer, inner retinal layer, rod-transducin- $\alpha, \alpha-$ enolase, and carbonic anhydrase II [15], with the latter two present in our patient.

Treatments for AIR include immunosuppressive agents aimed at decreasing circulating anti-retinal antibodies. Therapies include local or systemic corticosteroids, cyclosporine, mycophenolate mofetil, methotrexate, azathioprine, IVIG, and plasmapheresis $[8,9,16]$. There have also been reports of long-acting intravitreal fluocinolone acetonide implants being used for treatment of paraneoplastic and non-paraneoplastic AIR in patients refractory to or intolerant of systemic therapy [17]. More recently rituximab, an immunosuppressive agent targeting the CD20 protein on B lymphocytes, has been shown to be effective at stabilizing or improving clinical and laboratory markers of CAR, MAR, and npAIR in some patients $[18,19]$. This was true for our patient who despite initial improvement with prednisone and methotrexate, later progressed on these therapies and has shown GVF and OCT improvement after receiving rituximab infusions. Further followup is needed to evaluate for a sustained response to rituximab.

This report demonstrates that MGUS should be considered in the differential diagnosis of patients presenting with autoimmune retinopathy. Importantly, ocular symptoms may be the initial presentation of MGUS, and recognition of this may allow for earlier detection and initiation of monitoring for progression to $\mathrm{WM}$ or multiple myeloma. Ophthalmologists should consider rituximab therapy for MGUS-associated and other forms of AIR that have been refractory to other therapies.

\section{Abbreviations}

AIR: Autoimmune retinopathy; CAR: Cancer-associated retinopathy; ERG: Electroretinography; FA: Fluorescein angiography; GVF: Goldmann visual field; MAR: Melanoma-associated retinopathy; MGUS: Monoclonal gammopathy of undetermined significant; npAIR: Non-paraneoplastic autoimmune retinopathy; OCT: Optical coherence tomography; WM: Waldenström's macroglobulinemia

\section{Acknowledgements}

Kari Branham, MS, CGC assisted in genetic evaluation and testing of this patient.

\section{Authors' contributions}

EE analyzed and interpreted the clinical data and drafted the manuscript. GA acquired and interpreted clinical data and revised the manuscript. NK acquired and analyzed clinical data and revised the manuscript. AF acquired analyzed, and interpreted clinical data and substantively revised the manuscript. All authors read and approved the final manuscript.

Funding

AF is supported by a National Institutes of Health K12 Award (K12EY022299).

Availability of data and materials

The data from this study are available from the corresponding author on request. 


\section{Ethics approval and consent to participate}

Institutional Review Board approval was not required for this manuscript according to institutional guidelines. The patient consented to participate orally and in writing.

\section{Consent for publication}

Written informed consent was obtained from the patient for publication of this article and accompanying images. A copy of the written consent is available for review on request.

\section{Competing interests}

The authors declare they have no competing interests.

\section{Author details}

${ }^{1}$ Kellogg Eye Center, University of Michigan, 1000 Wall Street, Ann Arbor, M 48105, USA. ${ }^{2}$ Kresge Eye Institute, Wayne State University, 4717 St. Antoine, Detroit, MI 48201, USA.

Received: 21 May 2019 Accepted: 6 April 2020

Published online: 16 April 2020

\section{References}

1. Agarwal A, Ghobrial I. Monoclonal gammopathy of undetermined significance and smoldering multiple myeloma: a review of the current understanding of epidemiology, biology, risk stratification and management of myeloma precursor disease. Clin Cancer Res. 2013;19(5):985-94.

2. Kyle RA, Larson DR, Therneau TM, Dispenzieri A, Kumar S, Cerhan JR, et al. Long-term follow-up of monoclonal gammopathy of undetermined significance. N Engl J Med. 2018;378(3):241-9.

3. Saffra N, Rakhamimov A, Solomon WB, Scheers-Masters J. Monoclonal gammopathy of undetermined significance maculopathy. Can J Ophthalmol. 2013:48:e168-70.

4. Koo H, Oh DH, Chun YS, Kim JC. A case of crystalline keratopathy in monoclonal gammopathy of undetermined significance (MGUS). Korean J Ophthalmol. 2011;25(3):202-5.

5. Tzelikis PF, Laibson PR, Ribeiro MP, Rapuano CJ, Hammersmith KM, Cohen EJ. Ocular copper deposition associated with monoclonal gammopathy of undetermined significance: case report. Arq Bras Oftalmol. 2005;68:539-41.

6. Kyle RA, Therneau TM, Rajkumar SV, Larson DR, Plevak MF, Offord JR, et al. Prevalence of monoclonal gammopathy of undetermined significance. $\mathrm{N}$ Engl J Med. 2006;354:1362-9.

7. Van de Donk NW, Palumbo A, Johnsen HE, Engelhardt M, Gay F, Gregersen $\mathrm{H}$, et al. The clinical relevance and management of monoclonal gammopathy of undetermined significance and related disorders: recommendations from the European myeloma network. Haematologica. 2014;99(6):984-96.

8. Grange L, Dalal M, Nussenblatt RB, Sen HN. Autoimmune retinopathy. Am J Ophthalmol. 2014;157:266-72.

9. Heckenlively JR, Ferreyra HA. Autoimmune retinopathy: a review and summary. Semin Immunopathol. 2008;30:127-34.

10. Chan JW. Paraneoplastic retinopathies and optic neuropathies. Surv Ophthalmol. 2003;48:12-38.

11. Rahimy E, Sarraf D. Paraneoplastic and non-paraneoplastic retinopathy and optic neuropathy: evaluation and management. Surv Ophthalmol. 2013;58: 430-58

12. Liu Y, Magro C, Loewenstein Jl, Makar RS, Stowell CP, Dzik WH, et al. A man with paraneoplastic retinopathy plus small fiber polyneuropathy associated with Waldenström macroglobulinemia (lymphoplasmacytic lymphoma): insights into mechanisms. Ocul Immunol Inflamm. 2015;23:405-9.

13. Sen HN, Chan CC, Caruso RC, Fariss RN, Nussenblatt RB, Buggage RR. Waldenström's macroglobulinemia-associated retinopathy. Ophthalmology. 2004;111:535-9.

14. Shimanovsky A, Alvarez Argote J, Murali S, Dasanu CA. Autoimmune manifestations in patients with multiple myeloma and monoclonal gammopathy of undetermined significance. BBA Clin. 2016;6:12-8.

15. Grewal DA, Fishman GA, Jampol LM. Autoimmune retinopathy and antiretinal antibodies: a review. Retina. 2014;34:827-45.

16. Ferreyra HA, Jayasundera T, Khan NW, He S, Lu Y, Heckenlively JR. Management of autoimmune retinopathies with immunosuppression. Arch Ophthalmol. 2009;127:390-7.
17. DiLoreto DA, Williams Z, Khalifa YM. Autoimmune retinopathy treated with the fluocinolone acetonide intravitreal implant after intolerance to systemic immunosuppression. Invest Ophthalmol Vis Sci. 2014;55(13):2517.

18. Davoudi S, Ebrahimiadib N, Yasa C, Sevgi DD, Roohipoor R, Papavasilieou E, et al. Outcomes in autoimmune retinopathy patients treated with rituximab. Am J Ophthalmol. 2017;180:124-32.

19. Boudreault K, Justus S, Sengillo JD, Schuerch K, Lee W, Cabral T, et al. Efficacy of rituximab in non-paraneoplastic autoimmune retinopathy. Orphanet J Rare Dis. 2017;12:129.

\section{Publisher's Note}

Springer Nature remains neutral with regard to jurisdictional claims in published maps and institutional affiliations.
Ready to submit your research? Choose BMC and benefit from:

- fast, convenient online submission

- thorough peer review by experienced researchers in your field

- rapid publication on acceptance

- support for research data, including large and complex data types

- gold Open Access which fosters wider collaboration and increased citations

- maximum visibility for your research: over $100 \mathrm{M}$ website views per year

At $\mathrm{BMC}$, research is always in progress.

Learn more biomedcentral.com/submissions 